REVISTA ANDALUZA DE ANTROPOLOGÍA.

NÚMERO12:PATRIMONIOINMATERIAL:REDUCCIONISMOS, CONFLICTOSE INSTRUMENTALIZACIONES. INTANGIBLE CULTURAL HERITAGE: REDUCTIONISMS, CONFLICTS AND INSTUMENTALIZATIONS.

MARZO DE 2017

ISSN 2174-6796

[pp. 117-143]

http://dx.doi.org/10.12795/RAA.2017.12.06

Recibido: $18 / 10 / 2016$

Aceptado: 24/01/2017

\title{
EL PATRIMONIO INMATERIAL EN EL PAÍS VALENCIANO: UNA EXPLOSIÓN MUY TANGIBLE ${ }^{1}$ INTANGIBLE HERITAGE IN THE PAÍS VALENCIANO: A VERY TANGIBLE EXPLOSION
}

\section{Beatriz Santamarina \\ Universidad de Valencia}

\section{Resumen.}

En este artículo proponemos una aproximación a las políticas impulsadas, en los últimos años, sobre el patrimonio inmaterial valenciano. Para ello, en primer lugar, proponemos un breve marco interpretativo desde donde poder abordar el análisis que nos ocupa. La explosión de lo inmaterial se encuadra en las nuevas políticas patrimoniales desplegadas por la UNESCO, pero también responde al llamado tercer espíritu del capitalismo y a las transformaciones acaecidas en los sujetos/objetos patrimoniales. En segundo lugar, realizamos una aproximación al marco normativo del patrimonio cultural valenciano, para ver cómo ha encajado en su legislación lo inmaterial. En tercer lugar, tratamos de ver qué imagen se proyecta o se construye tras las activaciones patrimoniales de los bienes inmateriales en el País Valenciano. Desde nuestra consideración, resulta significativa, por un lado, la ubicación de los bienes escogidos en cuanto encapsuladores de lo que

1. Este trabajo se enmarca en el proyecto "El patrimonio cultural y natural en tiempos de crisis. Retos, adaptaciones y estrategias en contextos locales" (CSO2015-68611-R) financiado por el Ministerio de Economía y Competitividad. 
se entiende o se impone como patrimonio inmaterial; y, por otro, la estrategia política mostrada hasta el momento, que parece enfocarse hacia para el reconocimiento de bienes para la Lista de Patrimonio Cultural Inmaterial de la Humanidad. Por último, para cerrar este trabajo, realizamos una reflexión sobre el tipo de activaciones que se están produciendo y nos planteamos si la fetichización y mercantilización de lo inmaterial puede ser sorteada con la participación y la implicación de la comunidad.

\title{
Palabras clave.
}

Patrimonio inmaterial, País Valenciano, salvaguardia, políticas hegemónicas, regímenes patrimoniales

\begin{abstract}
.
In this article we propose an approximation to the policies implemented in the last few years on the Valenciano intangible heritage. To achieve this goal, first we propose a succinct interpretative framework to contribute to the analysis of patrimonial issues. The explosion of the intangible must be understood in the context of the heritage policies unfolded by UNESCO but also taking into account the so called "capitalism third spirit" and the transformations suffered by the patrimonial subjects/objects. Secondly, we analyze the Valencian normative cultural framework to understand how it has absorbed the intangible in its legislation. Thirdly, we highlight the image that is constructed and projected by the patrimonial activation of the PaísValencià intangible goods. The location of the chosen goods is extremely relevant as it has an impact on what is considered or imposed as intangible heritage. The political strategy used to date seems focused on achieving recognition by List of the Intangible Cultural Heritage of Humanity. Lastly, the article discusses if participation and community implication can counteract the fetishization and commodification that one observes in current processes of patrimonial implementation.
\end{abstract}

\section{Key words.}

Intangible heritage, País Valenciano, safeguarding, hegemonic policies, heritage regimes

\section{INTRODUCCIÓN}

Durante las dos últimas décadas, hemos asistido a un crecimiento exponencial del patrimonio cronológico, topográfico, categorial y conceptual (Heinich, 2009; Bendix, 2009). Éste ha sido numerosas veces señalado y afecta a cualquier distinción analítica patrimonial (cultural, natural e inmaterial), poniendo en tela de juicio la propia lógica de las activaciones patrimoniales como bienes escasos (García, 1998; Lacarrieu, 2008). El patrimonio colectivo se ha ido expandiendo con rapidez, durante los últimos 
cincuenta años, a la par que las instituciones, normativas, especialistas y publicaciones se multiplicaban, convirtiéndose en un potente fetiche metacultural (KirshenblattGimblett, 2004).

Desde distintas perspectivas y disciplinas, el análisis de la fábrica patrimonial ha permitido sacar a la luz su naturaleza compleja. Los procesos patrimoniales y la dimensión hegemónica del patrimonio se han convertido en el centro del debate y han producido, cada vez más, una interesante bibliografía (Smith, 2004; 2006; Goldman, 2001; Herzfeld, 2006; 2010; Smith y Akagawa, 2009; MacDonald, 2013; Franquesa, 2013; Harrison, 2013; Sánchez-Carretero, 2012; Bendix, Eggert y Peselmann, 2012; Del Marmól, 2012; Chaves, Montenegro y Zambrano, 2014; Del Mármol, Siniscalchi y Estrada, 2016). Hoy, lejos de ser visto como un áurea transcendental y como una realidad inmanente, el patrimonio ha sido desenmascarado como discurso autorizado (Smith, 2006) y como constructo político atravesado por la reinvención, el pasado y la identidad (Hobsbawm y Ranger, 1983; Lowenthal, 1985; Iniesta, 1994; Prats, 1997; Quintero, 2005; Hernández et al., 2005).

Partiremos aquí, por tanto, de considerar que no existen elementos con una cualidad intrínsecamente patrimonial (Kirshenblatt-Gimblett, 1998; Harvey, 2001; Smith, 2004; 2006) sino agentes patrimonializadores (Montenegro, 2010); y abordaremos el patrimonio como una categoría política, en la que se plasman distintos espacios de disputa ideológicos (Smith, 2006; Bendix, 2009; Bendix, Eggert and Peselmann, 2012; Chaves, Montenegro y Zambrano, 2014). Entendemos que su producción está articulada en un sistema global e institucionalizado de valores, generador de distinciones hegemónicas -transformadas en sentidos preceptivos y performativos- donde se escenifican tanto las distintas estrategias y agentes, como la estructura de la distribución asimétrica de sus capitales (Bourdieu, 1991, 1998; Comaroff y Comaroff, 1991; Herzfeld, 2004, 2006; 2010; Palumbo, 2010; Santamarina, 2013). La UNESCO, como la agencia por excelencia de la normativa patrimonial, ha jugado un papel fundamental en la construcción de taxonomías globales. En primer lugar, por su trabajo para la conservación/preservación patrimonial, entendiendo, como acabamos de señalar, que la conservación es connaturalmente política. Y en segundo lugar, por su labor para la definición y la constitución de categorías patrimoniales. Esto último es especialmente interesante porque del relato y enumeración de los diferentes patrimonios se desprenden trascendentales razones ideológicas y prácticas. Además, a través de su trabajo se hace evidente la exportación y colonización de modelos cognitivos y las nuevas formas de dominación postcoloniales.

Dicho esto, nos interesa señalar aquí que el patrimonio ha sufrido una transformación espectacular, de objetos y sujetos, de agentes y agencias (Ariño, 2002; Santamarina, 2005). De todas ellas nos interesa destacar la última novedad institucionalizada, la incorporación de lo inmaterial, con entidad propia, en la UNESCO (Convención para la 
Salvaguardia del Patrimonio Cultural Inmaterial, París, 2003). Más allá de toda la polémica conceptual, que rodea a lo inmaterial por su carácter procesual, y de los problemas teóricos-metodológicos derivados de su inclusión (Bouchenaki, 2003; Quintero, 2003; Van Zanten, 2004; Aikawa, 2004; Kurin, 2004; 2007; Lacarrieu, 2008; Bortolotto, 2011a; 2011b; Khaznadar, 2009; Hafstein, 2011; Bendix, 2011; Noyes, 2011; Del Malmól, Roigé y Estrada, 2011; Estrada y Del Mármol, 2014), en esta ocasión queremos reparar, en las formas en que puede ser interpretado este reconocimiento. Sin duda, esta admisión puede ser leída desde varias ópticas: como una compensación simbólica por parte de la UNESCO; como una muestra más de la transformación de la economía neoliberal con la llegada de la economía de los intangibles y las nuevas economías culturales; como una respuesta ante la demanda de lo 'auténtico'; y como una posibilidad de reafirmación de la heterogeneidad y las diferencias ante la reiterativa retórica de los peligros de la homogeneización y desterritorialización provocados por la globalización.

En cualquier caso, en este contexto, de hiperinflación patrimonial, de economía de los intangibles, y de desembarco de lo inmaterial, proponemos una aproximación al patrimonio inmaterial valenciano. Nos interesa, en concreto, reparar las activaciones que se han producido en este territorio. La mayoría de las declaratorias de patrimonio inmaterial se han sucedido a partir del 2010. En el conjunto del estado español ${ }^{2}$, el País Valenciano ostenta el mayor número de manifestaciones inscritas en la Lista Patrimonio Cultural Inmaterial de la Humanidad ${ }^{3}$ (Misteri d'Elx, Tribunal de las Aguas de la Huerta de Valencia, Centro de Cultura Tradicional-Museo Escolar de Pusol, la fiesta de la Mare de Déu de la Salut de Algemesí y el Espacio Cultural de la fiesta de las fallas valencianas)4. Cuenta con veinticinco bienes inmateriales declarados Bien de Interés cultural (BIC) y cinco Bienes de Relevancia Local Inmaterial. Esto denota el potencial del patrimonio inmaterial en el País Valenciano y el interés por poner en valor este patrimonio en los últimos años.

En este artículo, comenzamos con una sintética contextualización teórica como marco para interpretar el análisis de nuestro objeto. La irrupción de lo inmaterial se inserta en el nuevo régimen patrimonial abanderado por las agencias hegemónicas patrimoniales, en las propias transformaciones de los sujetos/objetos patrimoniales y en las nuevas lógicas

2. España cuenta con 15 bienes reconocidos en la Lista de Patrimonio Cultural Inmaterial de la Humanidad, situándose a la cabeza de los países europeos.

3. No contabilizamos las distinciones que afectan a todo el estado español y a otros países como la Cetrería, (2012) y la Dieta Mediterránea (2013).

4. Además, en 2011 se presentó la candidatura, junto con otras comunidades autónomas, “Tamboradas. Rituales de toque de tambor”. Ésta fue rechazada y está pendiente su nueva presentación a la UNESCO. 
de producción posfordistas. Tras dibujar las coordenadas interpretativas, realizaremos una aproximación al marco legal del patrimonio cultural valenciano, con el objeto de ver cómo se ha articulado lo inmaterial en el campo de lo preceptivo. Después analizaremos las activaciones patrimoniales de los bienes inmateriales en el País Valenciano, atendiendo a su localización, tipología y estatus normativo. Por último, cerraremos con algunas reflexiones a partir del análisis de las políticas patrimoniales de lo inmaterial vistas en este territorio.

\section{LA EXPLOSIÓN DE LO INMATERIAL}

La explosión de lo inmaterial, como venimos apuntando, responde a las nuevas políticas patrimoniales, pero también al llamado tercer espíritu del capitalismo y a los cambios producidos tanto en los sujetos como en los objetos patrimoniales. Su admisión ha traído pareja otras importantes mutaciones en la maquinaria patrimonial. De manera sintética, hemos pasado del énfasis en el objeto al acento en el proceso, de la distinción a la diferencia como marca, de la individualidad a la colectividad como sujeto, del genio creativo a la comunidad imaginada ${ }^{5}$, de la conservación y restauración a la salvaguardia y del eurocentrismo al globocentrismo.

Pero, más allá de todas estas transformaciones, su reconocimiento responde a varias consideraciones. En primer lugar, el desembarco de lo inmaterial puede ser visto como una compensación simbólica al 'olvido' de los otros patrimonios por parte de la UNESCO. Una reparación moral a las distinciones practicadas. Así como, también, una forma de compensar la distribución asimétrica de sus bienes culturales y naturales en el mapa patrimonial ${ }^{6}$. En segundo lugar, no puede pasarse por de alto que su reconocimiento responde a los cambios de la racionalidad de la economía neoliberal. La llegada del tercer espíritu del capitalismo, con la economía de los intangibles, ha transformado el engranaje de los valores. El acento en lo material ha sido desplazado a favor de lo inmaterial (Boltanski y Chiapello, 2002; Harvey, 2005; 2012; Comaroff y Comaroff, 2011). Y, en relación con esto último, debe contextualizarse la llamada 'inflación’ patrimonial. La demanda y la oferta de lo patrimonial han alcanzado cuotas elevadísimas impulsadas por la terciarización de la economía y la expansión del turismo (Nash, 1992; Esteve, 1983; MacCannell, 1999; West y Carrier, 2004). El valor de lo 'auténtico', como un añadido de diferenciación y como un plus frente a lo artificial, mueve hoy las mercancías glocalizadas

5. Hacemos aquí un uso particular del concepto de Anderson (1983). Para una aproximación al polémico e idealizado término de comunidad ver Bauman (2001).

6. Este discurso se puede ver explicito en la Global Strategy for a Representative, Balanced and Credible World Heritage List (http://whc.unesco.org/en/globalstrategy) o en el discurso del propio Koichiro Matsuura, director de la UNESCO entre 1999y 2009 (Aikawa, 2004). 
(Davallon, 2010; Frigolé, 2014; Santamarina y Moncusí, 2015). Si a esto le añadimos la obsesión en los relatos por la reafirmación de la heterogeneidad y las diferencias, frente a los procesos de homogeneización, desterritorialización e hibridación, se entenderá mejor el énfasis en salvaguardar las diferencias.

En este contexto, desde nuestro punto de vista, se torna pertinente hablar de explosión del patrimonio inmaterial, pareja a la sufrida por las categorías clásicas de clasificación patrimonial. Esta puede ser testada a través de una aproximación al patrimonio globalizado. Tras la Convención para la Salvaguardia del Patrimonio Cultural Inmaterial (París, 2003), la Lista Representativa del Patrimonio Cultural Inmaterial de la Humanidad (PCI) se activó en el 2006 y comenzó su andadura, en el 2008, cuando se nutrió de todos los bienes declarados como Obras Maestras del Patrimonio Oral e Intangible de la Humanidad. Las Obras Maestras, en sus sucesivas declaratorias cada dos años $(2001,2003$ y 2005), habían alcanzado el reconocimiento de noventa bienes. Es interesante, en este sentido, tener presente que el número de declaratorias se fue doblando en ese periodo, de diecinueve a veintitrés y de veintitrés a cuarenta y ocho. En el 2009, se ampliaba sumando a la misma otros setenta y ocho bienes (ese mismo año se declaraban doce bienes en Lista del Patrimonio Cultural que requiere medidas urgentes de salvaguardia) ${ }^{7}$ y, en el 2010, se incorporaban otros cuarenta y cuatro (más cuatro en lista de medidas urgentes). Este crecimiento exponencial demostraba su vitalidad y los resultados de los programas iniciados anteriormente por la UNESCO como el Funds-in-Trust for the Preservation and Promotion of the Intangible Cultural Heritage (1993).

A partir del 2011 y hasta el $2015^{8}$, el crecimiento se ha reducido y estabilizado y se han incluido una media de veintinueve bienes por año en la lista de PCI, una cifra nada desdeñable si se piensa en la complejidad burocrática de los expedientes que rodean a cualquier candidatura. Hoy, la Lista de Patrimonio Inmaterial de la Humanidad la conforman 356 bienes, pese a que todavía si los comparamos con la Lista de Patrimonio de la Humanidad de Bienes Culturales observamos un claro desequilibrio, dado que estos últimos doblan su número (725). La tendencia, de seguir así, parece indicar que en poco más de una década las listas estarán compensadas.

Pero, ¿Qué impacto ha tenido en la esfera de lo local la activación del patrimonio inmaterial globalizado? ¿De qué manera se ha articulado lo inmaterial en las políticas locales? ¿Han seguido la estela de lo establecido en la UNESCO? ¿Se han adaptado a las

7. No vamos a distinguir aquí la categoría de Mejores Prácticas de Salvaguardia.

8. En el 2011, 25; en el 2012, 34; en el 2013, 27; en el 2014, 35; y en el 2015, 23. En estos años, el número de bienes declarados en Lista del Patrimonio Cultural que requiere medidas urgentes de salvaguardia se ha elevado a 26. Para más información http://www.unesco.org/culture/ich/es/listas 
nuevas exigencias del mercado patrimonial? Para analizar todo ello, proponemos en lo que sigue, acercarnos a un caso concreto y ver de qué manera se plasma lo inmaterial en un territorio específico. Primero atenderemos a la legislación producida sobre el patrimonio valenciano colectivo, observando sólo a los aspectos que inciden en lo inmaterial, para pasar luego a lo sucedido en las declaratorias que se han concentrado, sobre todo, en la última década.

\section{LA NORMATIVA DEL PATRIMONIO CULTURAL VALENCIANO}

En el País Valenciano la Ley 4/1998, de 11 de junio, del Patrimonio Cultural Valenciano9, establecía la doctrina jurídica sobre el patrimonio valenciano. Por lo que respecta al patrimonio inmaterial, la normativa recogía en su descripción, dentro de la definición amplia de su objeto, que "forman parte del patrimonio cultural valenciano, en calidad de bienes inmateriales del patrimonio etnológico, las creaciones, conocimientos y prácticas de la cultura tradicional valenciana" (Articulo 1.3). Asimismo, cuando definía las clases de Bienes de Interés Cultural Valenciano en su capítulo III $^{10}$, especificaba sobre los bienes inmateriales de interés cultural: "Aquellas actividades, conocimientos, usos y técnicas que constituyen las manifestaciones más representativas y valiosas de la cultura y los modos de vida tradicionales de los valencianos serán declarados Bienes de Interés Cultural" (artículo 26) ${ }^{11}$. Y por lo que respecta a los Bienes de Relevancia Local se decía prácticamente lo mismo, excluyendo el 'más' y 'valiosas': "se incluirán en el Inventario General del Patrimonio Cultural Valenciano, como bienes inmateriales del patrimonio etnológico, aquellos conocimientos, técnicas, usos y actividades representativos de la cultura tradicional valenciana" (Artículo 55). La definición, como vemos, era genérica y no establecía categorías diferenciadas para el patrimonio inmaterial, en consonancia con otras legislaciones del momento en el estado español ${ }^{12}$. Y establecía de forma clara una jerarquización de los bienes (de más a menos).

9. LEY 4/1998, de 11 de junio, de la Generalitat Valenciana, del Patrimonio Cultural Valenciano (Diari Oficial núm. 3267 de 18.06.1998). Disponible en: http://www.dogv.gva.es/portal/ficha_disposicion_ pc.jsp?sig=1137/1998\&L=1

10. La normativa, sigue el modelo BIC de la legislación estatal, pero redefiniéndola. De este modo, distingue entre Bienes de Interés Cultural Valenciano, Bienes inventariados no declarados de interés cultural y Bienes inventariados no declarados del patrimonio cultural.

11. En esta ley se distinguía cuatro clases de BIC: bienes inmuebles, bienes muebles, documentos y obras bibliográficas, bienes inmateriales. Esta clasificación se ha mantenido en las modificaciones de la normativa, no así su contenido.

12. Nótese que era definido como "bienes inmateriales del patrimonio etnológico". Para una aproximación al tratamiento jurídico del patrimonio etnológico e inmaterial se puede acudir a Santamarina, Hernández y Moncusí (2008), Pérez Galán (2011) y Martinez (2011). 
Esta normativa fue modificada cinco años después por la Ley 7/2004, de 19 de octubre $^{13}$. Y en la misma se pueden ver reflejados los cambios a nivel internacional en la concepción del patrimonio colectivo. La puesta en marcha Programa de Obras Maestras del Patrimonio Oral e Intangible de la Humanidad, adoptado por el Consejo Ejecutivo de la UNESCO en 1998, suponía un paso firme hacia el reconocimiento del patrimonio inmaterial. La propia declaración del Misteri d'Elx, en el 2001, como Obra Maestra, y dos años más tarde, la Convención para la Salvaguarda del Patrimonio Cultural Inmaterial (París, 2003) fueron, sin duda, el contexto que propició la modificación de la ley valenciana. Recordemos que esta última convención equiparaba en calidad y carácter al patrimonio inmaterial con el material y el natural, al otorgarle una igualdad conceptual y un cuerpo jurídico (Santamarina, 2013). El patrimonio cultural inmaterial, quedaba definido, por la UNESCO, como los "usos, representaciones, expresiones, conocimientos y técnicas -junto con los instrumentos, objetos, artefactos y espacios culturales que les son inherentes- que las comunidades, los grupos y en algunos casos los individuos reconozcan como parte integrante de su patrimonio cultural. Este patrimonio cultural inmaterial, que se transmite de generación en generación, es recreado constantemente por las comunidades y grupos en función de su entorno, su interacción con la naturaleza y su historia, infundiéndoles un sentimiento de identidad y continuidad y contribuyendo así a promover el respeto de la diversidad cultural y la creatividad humana" (artículo 2.1). Se proponían cinco categorías para su inventariado y catalogación: 1 . Tradiciones y expresiones orales, incluido el idioma; 2 . Artes del espectáculo: música, danza y teatro; 3. Usos sociales, rituales y actos festivos; 4. Conocimientos y usos relacionados con la naturaleza y el universo; 5 . Técnicas artesanales tradicionales.

Volviendo a la modificación del 2004 de la ley valenciana, podemos observar, en su preámbulo, un claro giro en la consideración del patrimonio inmaterial. De hecho, la justificación para la actualización de la normativa del 1998 especificaba la necesidad de "ajustar la ley a las exigencias de una sociedad valenciana cada vez más concienciada de la necesidad de proteger y dinamizar su patrimonio cultural entendido como algo vivo que se incrementa permanentemente en la medida en que permanentemente se materializan expresiones culturales de una sociedad especialmente dinámica como la valenciana. Precisamente uno de los grupos de bienes que revelan tal viveza, dinamismo y su importancia cultural es el de los denominados bienes inmateriales o intangibles". Las modificaciones introducidas se articulaban sobre cuatros ejes, dos de ellos referidos

13. LEY 7/2004, de 19 de octubre, de la Generalitat, de Modificación de la Ley 4/1998, de 11 de junio, del Patrimonio Cultural Valenciano (Diari Oficial núm. 4867 de 21.10.2004). Disponible en: http://www. dogv.gva.es $/$ portal/ficha disposicion.jsp?id=26\&sig=4651/2004\&L=1\&url lista 
específicamente al patrimonio inmaterial ${ }^{14}$. El primero trataba de reforzar el patrimonio inmaterial con la introducción de varios artículos que lo definían de manera más específica Y el segundo hablaba sobre la puesta en valor de los bienes de interés cultural inmateriales, reconociendo la particularidad de este patrimonio "permiten que estos bienes no se conviertan en piezas de museo inanimadas, carentes de vida, lo que no sólo generaría su degradación, sino la pérdida de usos y costumbres que son parte de nuestro patrimonio inmaterial, en última instancia” (Preámbulo).

En cualquier caso, modificaba el artículo 1, ampliándose la definición y conformando un punto aparte en la redacción de la misma: "forman parte del patrimonio cultural valenciano, en calidad de bienes inmateriales del patrimonio etnológico, las creaciones, conocimientos y prácticas de la cultura tradicional valenciana. Asimismo, forman parte de dicho patrimonio como bienes inmateriales las expresiones de las tradiciones del pueblo valenciano en sus manifestaciones, musicales, artísticas, gastronómicas o de ocio, y en especial aquellas que han sido objeto de transmisión oral y las que mantienen y potencian el uso del valenciano" (artículo 1, 3). Y, además, se añadía, como otro punto: "Los bienes inmateriales de naturaleza tecnológica que constituyan manifestaciones relevantes o hitos de la evolución tecnológica de la Comunidad Valenciana son, así mismo, elementos integrantes del patrimonio cultural valenciano" (artículo 1.4) ${ }^{15}$. En el artículo 15, el objeto y contenido del inventario, se volvía a reincidir sobre la formulación del objeto ${ }^{16}$. Y en el artículo 26, sobre las clases de BIC, quedaba definido de la siguiente manera: "Bienes inmateriales. Pueden ser declarados de interés cultural las actividades, creaciones, conocimientos, prácticas, usos y técnicas representativos de la cultura tradicional valenciana, así como aquellas manifestaciones culturales que sean expresión de las tradiciones del pueblo valenciano en sus manifestaciones musicales, artísticas o de ocio, y en especial aquellas que han sido objeto de transmisión oral y las que mantienen y potencian el uso del valenciano. Igualmente podrán ser declarados de interés cultural los bienes inmateriales de naturaleza tecnológica que constituyan manifestaciones relevantes o hitos de la evolución tecnológica de la Comunidad Valenciana” (Artículo 26). Modificándose sustancialmente la categoría formulada en 1998. En este sentido, se

14. Los otros dos ejes estaban dedicados uno al reconocimiento del patrimonio informático y el otro a la necesidad de constituir fundaciones para dinamizar el patrimonio cultural de los valencianos.

15. Al respecto, Martínez (2011) considera que esto es una propuesta singular al identificar "un segundo dominio de los bienes del patrimonio inmaterial" (2011:131).

16. "Los bienes inmateriales del patrimonio etnológico, constituidos tanto por los conocimientos, técnicas, usos y actividades más representativos y valiosos de la cultura y las formas de vida tradicionales de los valencianos y valencianas, como por las expresiones de las tradiciones del pueblo valenciano en sus manifestaciones musicales, artísticas, gastronómicas o de ocio y, en especial, aquellas que han sido objeto de transmisión oral y las que mantienen y potencian el uso del valenciano" (Artículo 15). 
añadían las creaciones y las prácticas, se suprimían los calificativos "más" y "valiosas", se especificaban manifestaciones, se incorporaba la transmisión oral y la lengua del país y, por último, se incluían bienes de naturaleza tecnológica. Todo ello estaba más acorde con los cambios internacionales experimentados en su concepción ${ }^{17}$. Por otra parte, mudaba la forma de referirse al patrimonio inmaterial de los valencianos: si en 1998 la ley hablaba de "la cultura y los modos de vida tradicionales de los valencianos", en su modificación se decía "tradiciones del pueblo valenciano". Es interesante está matización porque, en la primera versión, se utilizaba una distinción decimonónica apegada al imaginario del folclore que implicaba una clara divisoria, mientras que en esta se desvinculaba, apareciendo 'tradiciones' como un lugar compartido y 'pueblo valenciano' como un espacio vertebrador de identidad. Aún así, la normativa valenciana no escapa a lo que ha sido señalado por Martínez: "la mayoría de las leyes autonómicas que regulan el patrimonio inmaterial están inspiradas por una noción folclórica del patrimonio etnográfico o etnológico" (2011: 131).

Posteriormente, la Ley 5/2007 de 9 de febrero ${ }^{18}$ modificaba, de nuevo, la Ley del Patrimonio Cultural Valenciano, con tres objetivos claros: establecer criterios para los Planes Especiales de Protección de los BICs, asentar los criterios en los procesos de restauración y completar la sistemática del Inventario General (preámbulo). Con respecto a esto último, se complementaba el inventario introduciendo la figura de Espacio Etnológico como una categoría más de BIC. De hecho, el artículo 26 volvía a cambiarse y en la clasificación de los Bienes inmuebles aparecía, por primera vez, el Espacio Etnológico junto con las categorías establecidas en 1998 ${ }^{19}$. Éste era definido como: "Construcción o instalación o conjunto de éstas, vinculadas a formas de vida y actividades tradicionales, que, por su especial significación sea representativa de la cultura valenciana" (Artículo 26).

Como vemos, el corpus normativo del patrimonio valenciano se ha ido adaptando a las transformaciones experimentadas en el constructo patrimonial. Es probable que, a raíz de la Ley 10/2015 de 26 de mayo, para la salvaguardia del Patrimonio Cultural Inmaterial ${ }^{20}$ del estado español se vuelvan a introducir cambios. En la misma se recogía

17. Para una aproximación sobre estos cambios se puede acudir a Santamarina (2013).

18. LEY 5/2007, de 9 de febrero, de la Generalitat, de modificación de la Ley 4/1998, de 11 de junio, del Patrimonio Cultural Valenciano (Diari Oficial núm. 5449 de 13.02.2007). Disponible en: http://www. dogv.gva.es/portal/ficha_disposicion.jsp?id=24\&sig=1944/2007\&L=1\&url_lista

19. El sistema utilizado era el de categorías y siguiendo la normativa estatal, se recogían las siguientes categorías: Monumento, Conjunto Histórico, Jardín Histórico, Sitio Histórico, Zona Arqueológica, Zona Paleontológica y Parque Cultural.

20. Disponible en: https://www.boe.es/boe/dias/2015/05/27/pdfs/BOE-A-2015-5794.pdf 
que "Tendrán la consideración de bienes del patrimonio cultural inmaterial los usos, representaciones, expresiones, conocimientos y técnicas que las comunidades, los grupos y en algunos casos los individuos, reconozcan como parte integrante de su patrimonio cultural, y en particular: a) Tradiciones y expresiones orales, incluidas las modalidades y particularidades lingüísticas como vehículo del patrimonio cultural inmaterial; así como la toponimia tradicional como instrumento para la concreción de la denominación geográfica de los territorios; b) artes del espectáculo; c) usos sociales, rituales y actos festivos; d) conocimientos y usos relacionados con la naturaleza y el universo; e) técnicas artesanales tradicionales; f) gastronomía, elaboraciones culinarias y alimentación; g) aprovechamientos específicos de los paisajes naturales; h) formas de socialización colectiva y organizaciones; i) manifestaciones sonoras, música y danza tradicional". Como vemos, frente a las cinco categorías de clasificación propuestas por la UNESCO en el 2003, en la normativa estatal se diferencian hasta nueve ${ }^{21}$. Es probable, en este sentido, que en el futuro la legislación valenciana adopte y adapte también alguna clasificación.

\section{EL PATRIMONIO INMATERIAL EN EL PAÍS VALENCIANO}

Si atendemos, bajo lo que dictan las normativas, a lo declarado en el territorio valenciano podemos ver cómo se dibujan algunos de sus rasgos. Como ya hemos indicado tiene cinco bienes inscritos en la Lista Patrimonio Cultural Inmaterial de la Humanidad (Tabla 1). Los cinco se encuentran ubicados en los centros urbanos del País Valenciano (Valencia, Elche y Algemesí). Por otro lado, tres estarían declaradas bajo distintas categorías que podríamos agrupar como rituales y actos festivos (categoría 3, aunque en las declaratorias se contemplen más $)^{22}$, la otra podría considerarse como una práctica local de derecho consuetudinario (categorías 1, 3, 4 y 5) y una, el Centro de Pusol, es una buena práctica de salvaguardia.

\begin{tabular}{|l|l|l|}
\hline Año & Bien & Provincia \\
\hline 2001 & Misteri d'Elx & Alicante \\
\hline
\end{tabular}

21. Para ver los problemas derivados de esta ley se puede acudir a Carrera (2015).

22. Ya ha sido comentada la dificultad de establecer categorías entre el patrimonio inmaterial a la hora de clasificar prácticas. En la lista de la UNESCO, en el 75\% de bienes reconocidos "su inclusión en la lista responde a más de una categoría de las cinco que se proponen" (Santamarina, 2013:279). En el caso del Misteri d'Elx fue incluido bajo las categorías 2 y 3, los Tribunales de Regantes en las categorías 1, 3, 4 y 5; y La Festa de la Mare de Déu de la Salut d’Algemesí bajo las categorías 1, 2, 3 y 5. 


\begin{tabular}{|l|l|l|}
\hline 2009 & $\begin{array}{l}\text { Tribunales de regantes del Mediterráneo español: el Consejo } \\
\text { de Hombres Buenos de la Huerta de Murcia y el Tribunal de } \\
\text { las Aguas de la Vega de Valencia }\end{array}$ & Valencia \\
\hline 2009 & $\begin{array}{l}\text { El proyecto del Centro de Cultura Tradicional-Museo Escolar } \\
\text { de Pusol }\end{array}$ & Alicante \\
\hline 2011 & La Festa de la Mare de Déu de la Salut d’Algemesí & Valencia \\
\hline 2016 & Espacio Cultural de las Fallas & Valencia \\
\hline
\end{tabular}

Tabla 1. Lista Representativa del Patrimonio Cultural Inmaterial y Registro de las buenas prácticas de la salvaguardia de la UNESCO en la CV. Elaboración propia. Fuente: UNESCO.

Pero atendamos ahora a los Bienes de Interés Cultural Inmaterial (BICI) declarados en el País Valenciano. Entre los bienes inmateriales declarados BIC, dos están resueltos para todo el territorio valenciano (Tabla 2) por ser prácticas que se extienden por todo él, el juego de pelota (pilota valenciana) y el patrimonio normativo de la lengua (normes de Castelló). En este sentido, pese a que la práctica del toque de campanas también se encuentra extendida por todo el país, la declaración como BICI se resolvió mediante un único decreto que distinguía el toque en dos iglesias de Castellón y en dos de Valencia ${ }^{23}$, por eso hemos contabilizado, en lo que sigue, el toque de campanas como cuatro bienes cuando los computamos a un territorio y como un solo bien cuando analizamos la tipología de bienes.

\begin{tabular}{|l|l|l|}
\hline Año & Bien & Decreto \\
\hline 2014 & La pilota valenciana & $\begin{array}{l}\text { Decreto } 142 / 2014, \text { de } 5 \text { de septiembre } \\
\text { del Consell, } 7355 \text { de } 08.09 .2014\end{array}$ \\
\hline 2016 & Les Normes de Castelló & $\begin{array}{l}\text { Decreto 189/2016, de } 16 \text { de diciembre, } \\
\text { del Consell, publicado en el DOCV n } \\
\text { 7943 de 23.12.2016 }\end{array}$ \\
\hline
\end{tabular}

Tabla 2. Bienes de Interés Cultural Inmaterial para todo el ámbito del País Valenciano. Elaboración propia. Fuente: Dirección General de Patrimonio/DOCV

23. "Se declara Bien de Interés Cultural Inmaterial los toques manuales de campanas en la Iglesia Parroquial de la Asunción de Ntra. Sra. de Albaida, en el campanar de la Vila de Castellón de la Plana, en la Santa Iglesia Catedral Basílica de Santa María de la Asunción de Segorbe y en la Santa Iglesia Catedral Basílica Metropolitana de Santa María de Valencia" (Artículo 1. Decreto 111/2013, de 1 de agosto). 
El resto de los veintitrés bienes inmateriales BIC se distribuye de la siguiente manera: la provincia de Alicante tiene declarados cinco bienes (Tabla 3). Cuatro se ubican en zonas urbanas, ciudades capitales de comarca (dos en la ciudad de Alicante, capital de provincia y capital de la Comarca de Campo de Alicante y dos en la ciudad de Alcoy capital de la Comarca de la Hoya de Alcoy) y uno se encuentra en zona rural, una pequeña población del interior de la comarca de Campo de Alicante.

\begin{tabular}{|l|l|l|}
\hline Año & Bien & Decreto \\
\hline 2002 & Belén de Tirisiti de Alcoi & $\begin{array}{l}\text { Decreto 192/2002 de 26 de noviembre } \\
\text { del consell, publicado en el DOGV n } \\
4389 \text { de 29/11/2002. }\end{array}$ \\
\hline 2005 & Misteri d'Elx & $\begin{array}{l}\text { Ley 13/2005, de 22 de diciembre, de la } \\
\text { Generalitat, publicado en el DOGV n } \\
5166 \text { de 30/12/200524 }\end{array}$ \\
\hline 2011 & $\begin{array}{l}\text { Cabalgata de Reyes Magos de } \\
\text { Alcoi }\end{array}$ & $\begin{array}{l}\text { Decreto 199/2011 de 23 de diciembre } \\
\text { del consell, publicado en el DOCV n } \\
\text { 6678, de 26/12/2011 }\end{array}$ \\
\hline 2012 & $\begin{array}{l}\text { Pa Beneit de la Torre de les } \\
\text { Maçanes }\end{array}$ & $\begin{array}{l}\text { Decreto 153/2014, de 26 de septiembre, } \\
\text { del Consell, publicado en el DOCV n } \\
7370 \text { de 29/09/2014 }\end{array}$ \\
\hline 2014 & $\begin{array}{l}\text { Fogueres de Sant Joan } \\
\text { d'Alacant }\end{array}$ & $\begin{array}{l}\text { Decreto 222/2014, de 19 de diciembre, } \\
\text { del Consell, publicado en el DOCV } \mathrm{n}^{\circ} \\
7428 \text { de 22.12.2014 }\end{array}$ \\
\hline
\end{tabular}

Tabla 3. Bienes de Interés Cultural Inmaterial declarados en la provincia de Alicante. Elaboración propia. Fuente: Dirección General de Patrimonio/DOCV

La provincia de Castellón, por su parte, tiene declarados siete bienes inmateriales declarados BIC (tabla 4). Dos de ellos, como ya hemos anotado, son toques de campana.

24. En el caso del Misteri d'Elx la declaración de BIC se hizo a través de una ley. Tal y como se recogía en su preámbulo esto era debido a su singularidad: "La Festa o Misteri d'Elx es un tesoro cultural del pueblo de Elche y una de las joyas más preciadas del patrimonio cultural valenciano, como prueban su declaración como Monumento Nacional el año 1931, o su inclusión, en el año 2001, en la primera Proclamación de las Obras Maestras del Patrimonio Oral e Inmaterial de la Humanidad por la Organización de las Naciones Unidas para la Educación, la Ciencia y la Cultura (UNESCO)". En este sentido, es reseñable señalar que la declaración del Misteri de’Elx como monumento nacional por la II República (Gazeta de Madrid n²59, 16 septiembre de1931) fue el primer bien inmaterial reconocido en el estado español. 
En este caso, la distribución se reparte del siguiente modo: cuatro de ellos se ubican en zonas urbanas, ciudades todas ellas capitales de comarca (dos se encuentran en la ciudad de Segorbe en la comarca del Alto Palancia fronteriza con la provincia de Valencia, uno en Alcora en la comarca del Alcalatén y uno en Castellón de la Plana, capital de provincia y capital de la Comarca de la Plana Alta) y los otros tres están en zonas rurales montañosas del interior (uno está en una pequeña población, el Forcall, que se encuentra en la Comarca de Los Puertos de Morella, en esa misma comarca, en población rural de Morella se encuentra otro y el tercero se distribuye entre dos pequeñas poblaciones, Les Useres y Culla, de las comarcas del Alcatén y Alto Maestrazgo respectivamente).

\begin{tabular}{|c|c|c|}
\hline Año & Bien & Decreto \\
\hline 2011 & $\begin{array}{l}\text { Entrada de Toros y Caballos } \\
\text { de Segorbe }\end{array}$ & $\begin{array}{l}\text { Decreto 6/2011 de } 4 \text { de febrero del } \\
\text { Consell, publicado en el DOCV } n^{\circ} \\
6454 \text {, de 07/02/2011. }\end{array}$ \\
\hline 2012 & La Santantonada de Forcall & $\begin{array}{l}\text { Decreto } 10 / 2012 \text { de } 5 \text { de enero del } \\
\text { Consell, publicado en el DOCV } n^{\circ} \\
6688 \text {, de } 10 / 01 / 2012\end{array}$ \\
\hline 2012 & $\begin{array}{l}\text { Tamborada de la Rompida de } \\
\text { la Hora de l'Alcora }\end{array}$ & $\begin{array}{l}\text { Decreto } 11 / 2012 \text { de } 13 \text { de enero del } \\
\text { Consell, publicado en el DOCV n }{ }^{\circ} 6692 \\
\text { de } 16 / 01 / 2012 \text {. }\end{array}$ \\
\hline 2013 & $\begin{array}{l}\text { El Toc Manual de Campanes } \\
\text { en el Campanar de la Vila de } \\
\text { Castelló de la Plana }\end{array}$ & $\begin{array}{l}\text { Decreto } 111 / 2013 \text {, de } 1 \text { de agosto, del } \\
\text { Consell, publicado en el DOCV n }{ }^{\circ} 7082 \\
\text { de 5/08/2013 }\end{array}$ \\
\hline 2013 & $\begin{array}{l}\text { El Toc Manual de Campanes } \\
\text { en la Santa Iglesia Catedral } \\
\text { Basílica de Santa María de la } \\
\text { Asunción de Segorbe }\end{array}$ & $\begin{array}{l}\text { Decreto } 111 / 2013 \text {, de } 1 \text { de agosto, del } \\
\text { Consell, publicado en el DOCV n } 7082 \\
\text { de } 5 / 08 / 2013\end{array}$ \\
\hline 2016 & $\begin{array}{l}\text { Las peregrinaciones por } \\
\text { Camins del Penyagolosa: Les } \\
\text { Useres Culla }\end{array}$ & $\begin{array}{l}\text { Decreto 53/2016, de } 29 \text { de abril, del } \\
\text { Consell publicado en el DOCV n }{ }^{\circ} 7773 \\
\text { de } 03.05 .2016\end{array}$ \\
\hline
\end{tabular}




\begin{tabular}{|l|l|l|}
\hline 2016 & Sexenni de Morella & $\begin{array}{l}\text { Decreto 21/2016, de 26 de febrero, del } \\
\text { Consell, publicado en el DOCV n } \\
\text { o } 7733 \\
\text { de 03.03.2016 }\end{array}$ \\
\hline
\end{tabular}

Tabla 4. Bienes de Interés Cultural Inmaterial declarados en la provincia de Castellón. Elaboración propia. Fuente: Dirección General de Patrimonio/DOCV

Por último, en la provincia de Valencia hay declarados once (tabla 5). Puede considerarse que se todos ellos encuentran en zonas urbanas. Siete están en la ciudad de Valencia, capital de la comunidad y de la provincia. Uno se encuentra en la ciudad de Alzira, capital de la comarca de la Ribera Alta, y otro en la ciudad de Algemesí, también en la Ribera Alta y a escasos kilómetros de la anterior. Alzira comparte, con Xàtiva, Gandia, Sueca y Torrent, otra distinción, en este caso todas las ciudades son capitales de comarca (La Costera, La Safor, Ribera Baja y Huerta Oeste). Por último, hay uno que hemos clasificado como urbano: Albaida. Podríamos considerarlo en una zona rural ${ }^{25}$, pero, en realidad, la localidad tiene una población intermedia y ha venido desarrollando actividades industriales lo que le acercaría más a áreas urbanas. Nos interesa destacar en cualquier caso, que este municipio en la comarca de la Vall d'Albaida tiene una población bastante superior a las localidades de las zonas rurales antes señaladas ${ }^{26}$.

\begin{tabular}{|l|l|l|}
\hline Año & Bien & Decreto \\
\hline 2006 & $\begin{array}{l}\text { Tribunal de las aguas de la } \\
\text { Vega de Valencia }\end{array}$ & $\begin{array}{l}\text { Decreto 73/2006 de 26 de mayo del } \\
\text { Consell, publicado en el DOCV n } 5269 \\
\text { de 30/05/2006 }\end{array}$ \\
\hline 2010 & $\begin{array}{l}\text { Solemnidad del Corpus } \\
\text { Christide Valencia }\end{array}$ & $\begin{array}{l}\text { Decreto 92/2010 de 28 de mayo del } \\
\text { Consell, publicado en el DOCV n } \\
6280, \text { de 02/06/2010. }\end{array}$ \\
\hline
\end{tabular}

25. Sabiendo lo problemático que es definir el concepto ciudad cualitativa y cuantitativamente, la divisoria practicada por el INE que considera ciudades a los núcleos urbanos por encima de los 10.000 habitantes y a las poblaciones intermedias aquellas que tienen habitantes entre los de 2000 y 10000 . Este criterio cuantitativo se complementa con las actividad productiva de la población, si el $40 \%$ no es agrario se puede considerar ciudad.

26. En Castellón y Alicante los bienes declarados en zonas rurales se asientan en núcleos con muchos menos habitantes que los seis mil que alcanza Albaida. Todos salvo Morella (con una población de 2600) están por debajo de los 1000 habitantes. 


\begin{tabular}{|c|c|c|}
\hline 2010 & $\begin{array}{l}\text { La Festa de la Mare de Déu de } \\
\text { la Salut d'Algemesí }\end{array}$ & $\begin{array}{l}\text { Decreto } 117 / 2010 \text { de } 27 \text { de agosto del } \\
\text { Consell, publicado en el DOCV no } 6345 \\
\text { de } 01 / 09 / 2010 \text {. }\end{array}$ \\
\hline 2012 & Tamborada d'Alzira & $\begin{array}{l}\text { Decreto } 11 / 2012 \text { de } 13 \text { de enero del } \\
\text { Consell, publicado en el DOCV n }{ }^{\circ} 6692 \\
\text { de } 16 / 01 / 2012\end{array}$ \\
\hline 2012 & $\begin{array}{l}\text { Las fiestas de las fallas } \\
\text { valencianas }\end{array}$ & $\begin{array}{l}\text { Decreto 44/2012, de } 9 \text { de marzo del } \\
\text { Consell, publicado en el DOCV n }{ }^{\circ} 6732 \\
\text { de } 12 / 03 / 2012\end{array}$ \\
\hline 2013 & $\begin{array}{l}\text { Los Toques Manuales de } \\
\text { Campanas en la iglesia } \\
\text { parroquial de la Asunción de } \\
\text { Ntra. Sra. de Albaida }\end{array}$ & $\begin{array}{l}\text { Decreto } 111 / 2013 \text {, de } 1 \text { de agosto, del } \\
\text { Consell, publicado en el DOCV n }{ }^{\circ} 7082 \\
\text { de 5/08/2013 }\end{array}$ \\
\hline 2013 & $\begin{array}{l}\text { Los } \text { Toques } \\
\text { de Campanas en Santa } \\
\text { Iglesia Catedral Basílica } \\
\text { Metropolitana de Santa María } \\
\text { de Valencia }\end{array}$ & $\begin{array}{l}\text { Decreto } 111 / 2013 \text {, de } 1 \text { de agosto, del } \\
\text { Consell, publicado en el DOCV n }{ }^{\circ} 7082 \\
\text { de 5/08/2013 }\end{array}$ \\
\hline 2015 & $\begin{array}{l}\text { Representació dels miracles o } \\
\text { milacres de Sant Vicent Ferrer. }\end{array}$ & $\begin{array}{l}\text { Decreto 43/2015, de } 10 \text { d'abril, del } 7503 \\
\text { de 13.04.2015 }\end{array}$ \\
\hline 2015 & $\begin{array}{l}\text { La fiesta de las Fallas de } \\
\text { Xàtiva, Gandia, Sueca, Alzira } \\
\text { y Torrent }\end{array}$ & $\begin{array}{l}\text { Decreto 225/2015, de } 4 \text { de diciembre, } \\
\text { del Consell, publicado en el DOCV no } \\
7675 \text { de } 10.12 .2015\end{array}$ \\
\hline 2016 & $\begin{array}{l}\text { Procesión cívica del Nou } \\
\text { d'Octubre en Valencia }\end{array}$ & $\begin{array}{l}\text { Decreto } 127 / 2016 \text {, de } 7 \text { de octubre del } \\
\text { Consell, publicado en el DOCV no } 7892 \\
\text { de } 09 / 10 / 2016\end{array}$ \\
\hline 2016 & $\begin{array}{l}\text { Actividades tradicionales de la } \\
\text { Albufera de Valencia }\end{array}$ & $\begin{array}{l}\text { Decreto } 164 / 2016 \text {, de } 4 \text { de noviembre, } \\
\text { del Consell, publicado en el DOCV no } \\
7917 \text { (15.11.2016) }\end{array}$ \\
\hline
\end{tabular}

Tabla 5. Bienes de Interés Cultural Inmaterial declarados en la provincia de Valencia. Elaboración propia. Fuente: Dirección General de Patrimonio/DOCV 
Por lo que respecta a los Bienes de Relevancia Local Inmaterial (BRLI) (tabla 6), uno de ellos afecta a todo el País Valenciano, tres están en la provincia de Valencia y uno en la provincia de Alicante. De los cuatro, tres son de ámbito urbano (dos se ubican en la ciudad de Gandía, capital de la comarca de La Safor y uno en la ciudad alicantina de Crevillent en la comarca del Bajo Vinalopó) y uno rural, que se encuentra en una pequeña población del interior de la Comarca de Los Serranos.

\begin{tabular}{|l|l|l|l|}
\hline 2010 & $\begin{array}{l}\text { Las Embajadas de Moros y } \\
\text { Cristianos de Crevillent }\end{array}$ & $\begin{array}{l}\text { Orden 32/2010, de 14 de } \\
\text { julio (Diari Oficial no 6359 } \\
\text { de 21.09.2010) }\end{array}$ & Alicante \\
\hline $\begin{array}{l}\text { La Tradición Musical Popular } \\
\text { Valenciana materializada por la } \\
\text { Sociedades Musicales (Bandas } \\
\text { de Música de la Comunitat } \\
\text { Valenciana) }\end{array}$ & $\begin{array}{l}\text { Orden 1/2011, de 12 de } \\
\text { julio (Diari Oficial no 6571 } \\
\text { de 22.07.2011) }\end{array}$ & País \\
Valenciano & La Mojiganga de Titaguas & $\begin{array}{l}\text { Orden 3/2012, de 23 de } \\
\text { enero (Diari Oficial n. 6713 } \\
\text { de 14.02.2012) }\end{array}$ & Valencia \\
\hline 2012 & El Tío de la Porra de Gandia & $\begin{array}{l}\text { Orden 22/2012, de 20 de } \\
\text { junio (Diari Oficial no 6816 } \\
\text { de 11.07.2012) }\end{array}$ & Valencia \\
\hline 2012 & El Canto de la Sibila de Gandia & $\begin{array}{l}\text { Resolución de 21 de } \\
\text { diciembre de 2012 } \\
\text { (Diari Oficial no 6929 de } \\
24.12 .2012)\end{array}$ & Valencia \\
\hline
\end{tabular}

Tabla 6. Bienes de Relevancia Local Inmaterial declarados en el País Valenciano. Elaboración propia. Fuente: Dirección General de Patrimonio/DOCV 
Por último, habría que tener en consideración lo ocurrido con los Festejos taurinos tradicionales declarados BICI, con Ley 6/2015, de 2 de abril, de Reconocimiento, Protección y Promoción de las Señas de Identidad del Pueblo Valenciano ${ }^{27}$. Esta Ley, en su Disposición adicional única, declaraba "los festejos taurinos tradicionales de la Comunitat Valenciana (bous al carrer) como patrimonio cultural inmaterial valenciano".

A través de esta vía, excepcional, a medida y usurpando competencias a la Direcció General de Patrimoni Cultural, los bous al carrer sorteaban el procedimiento establecido para cualquier expediente. En realidad, la historia se remontaba tres años atrás cuando, en enero del 2012, se hacía vigente la prohibición de las corridas de toros en Catalunya ${ }^{28}$. La respuesta del gobierno valenciano ${ }^{29}$ no se hizo esperar y el Boletín Oficial del Estado (BOE) publicaba el 14 de marzo del 2012 la "Resolución de 20 de febrero de 2012, de la Conselleria de Turismo, Cultura y Deporte, por la que se incoa expediente de declaración de bien de interés cultural inmaterial, a favor de los espectáculos taurinos «las corridas de toros» y los festejos taurinos tradicionales «els bous al carrer» en todo el ámbito territorial de la Comunitat Valenciana”. Se iniciaba así el procedimiento para declararlos BICI, siguiendo la normativa vigente que establece la necesidad de dos informes favorables, sobre un máximo de tres, de las instituciones culturales que la ley valenciana de patrimonio establece como consultivas. El problema vino con los informes de los expertos para la tramitación de su declaración. La Academia de Bellas Artes de San Carlos se pronunció en contra de la declaratoria. El Consell Valencià de Cultura, pese a que la comisión correspondiente de dicha institución realizó un informe contrario a la declaratoria, se manifestó a favor por silencio administrativo ${ }^{30}$. El desempate, requerido por la ley, vino de la mano de la Universitat de València que se pronunció en contra de su declaración. Visto el resultado, la Generalitat buscó una vía alternativa para poder lograr su declaratoria y respaldar la posición mantenida por su partido tras lo ocurrido en Catalunya. Así, saltándose los cauces legales para el proceso aprobó su declaración,

27. No entramos aquí en los debates abiertos sobre las señas de identidad del País Valenciano, baste señalar que han sido objeto de enfrentamientos constantes entre las distintas fuerzas políticas.

28. Dos años antes el Parlamento de Catalunya, el 28 de julio de 2010, había aprobado su abolición a partir de una Iniciativa Legislativa Popular.

29. El gobierno contaba con la mayoría absoluta del Partido Popular (PP).

30. El Pleno del Consell Valencià de Cultura, en manos del PP, votó en contra del informe emitido por la comisión, en una jugada política que se resolvió por la vía del silencio administrativo (lo que se traducía en una posición favorable). 
aunque la misma apenas estuvo unos meses vigente. La polémica ley sobre las señas de identidad Valenciana fue derogada, en enero del 2016, por el actual gobierno valenciano ${ }^{31}$, anulándose la declaración de BICI de los festejos taurinos ${ }^{32}$.

Volviendo a nuestro análisis, y teniendo presente que los procesos de patrimonialización siempre conllevan, de un lado, la jerarquización, clasificación, selección, prescripción, enumeración y categorización sobre aquello que es digno o no de considerarse; y de otro, la intervención de diferentes agentes que le confieren legitimidad, veamos qué rasgos se dibujan en la activación de los BICI y BRIL en el País Valenciano. Si atendemos a la distribución de los bienes, podemos observar un claro desequilibrio territorial. La provincia de Valencia ostenta once bienes declarados BICI y tres BRLI. Castellón le sigue con siete BIC y ningún BRLI. Y finalmente, Alicante cuenta tan sólo con cinco BICI y un BRLI. Asimismo, si atendemos a su distribución por comarcas, de las treinta y cuatro en total en las que se divide el país, solo la mitad, diecisiete, están representadas. Bien es cierto que estamos tratando de demarcaciones administrativas y políticas y no culturales, pero tampoco parece existir en las declaratorias una propuesta por áreas culturales representativas. En este sentido, sorprende ver en el mapa concentraciones y grandes vacíos. De igual modo, como hemos querido reflejar a lo largo de la presentación de los bienes, la mayoría de ellos están ubicados en urbes del País Valenciano. En las ciudades más grandes se concentran más de la mitad de los bienes declarados y, si sumamos los declarados en las capitales de comarca o núcleos urbanos, tres tercios se sitúan en territorio urbano. En toda la lista, tanto de BICI como de BRLI, sólo existen declarados cinco bienes en poblaciones rurales (una en la provincia de Valencia, una en Alicante y tres en Castellón) ${ }^{33}$.

La lista de las declaratorias de los BICI y BRLI parece no responder a ningún criterio espacial, aludiendo quizás más a cuestiones políticas y prácticas. Por una parte, al interés por impulsar futuras candidaturas para la Lista Patrimonio Cultural Inmaterial de la Humanidad o por cuestiones de 'urgencia' política de índole identitaria (Bous al Carrer, Processó civica del Nou d'Octubre Valencia y les Normes de Castelló). Y, por otra, a la estructura centralista de las instituciones y a la capacidad administrativa para elevar el expediente de declaración a la Direcció General de Patrimoni Cultural. Pese a que el

31. Formado por una coalición de izquierdas entre el Partido Socialista, Compromís y Podemos.

32. Ley 1/2016, de 26 de enero, de derogación de la Ley 6/2015, de 2 de abril, de la Generalitat, de Reconocimiento, Protección y Promoción de las Señas de Identidad del Pueblo Valenciano.

33. Esta imagen sorprende si pensamos en el imaginario occidental y en la identificación del patrimonio inmaterial con el folclore (la cultura popular) que se sitúa en los pueblos. También es curioso porque proyecta una imagen de donde está el territorio más rural de los valencianos. 
trámite administrativo es relativamente sencillo (se deben rellenar unas fichas) no se cuenta en las administraciones locales con personal suficiente para realizar este trabajo. Además, no existe ningún soporte técnico, desde la administración de la Generalitat, que pueda subsanar este desequilibrio. En este sentido, es interesante anotar que, en 2012, se constituyó una Comisión de trabajo de Patrimonio Inmaterial, dentro de la Direcció General de Patrimoni Cultural, para la resolución de los expedientes que no tuvo ni continuidad ni reconocimiento institucional. Recientemente, en junio del 2016, se ha constituido por la Orden 19/2016 una Comisión Técnica para el Estudio e Inventario del Patrimonio Inmaterial para funciones asesoras y consultivas ${ }^{34}$. De cualquier manera, lo que es importante de subrayar es que no ha habido ninguna estrategia en lo referente al patrimonio inmaterial, salvo un interés político puntual por activar algunos bienes. Esta falta de estrategia responde a su vez, a una falta de interés por este tipo de patrimonio frente al patrimonio material. Dentro de las políticas de patrimonio sigue dominando la visión decimonónica, dando mayor peso y valor al patrimonio artístico e histórico. Esto se traduce en falta de presupuestos y en falta de personal destinado al patrimonio inmaterial, de hecho en estos momentos hay pendientes de resolver más de cuarenta expedientes ${ }^{35}$.

Si prestamos atención a los bienes inmateriales declarados, los criterios parecen responder a la belleza o estética de su factura y a la espectacularidad de su puesta en escena. Aun sabiendo el riesgo de clasificarlos en una sola categoría ${ }^{36}$, y siguiendo la propuesta de la UNESCO, podemos decir, que de los veinticinco ${ }^{37}$, veintiuno son usos sociales, rituales o actos festivos. Los otros cuatro se podrían repartir del siguiente modo, un bien asociado a conocimientos y uso de la naturaleza (tribunal de las aguas), dos técnicas artesanales (toque campanas y actividades Albufera), uno a un arte de espectáculo (música) y otro a una expresión oral (lengua). Esto nos remite a una imagen clara de lo que se percibe como patrimonio inmaterial vinculado a lo festivo y a lo folclórico, siguiendo con una continuidad histórica abierta en el XIX.

34. ORDEN 19/2016, de 1 de junio, de la Conselleria de Educación, Investigación, Cultura y Deporte, por la que se constituye una comisión técnica para el estudio e inventario del patrimonio inmaterial (DOGV $\left.\mathrm{n}^{\circ} 7800,8 / 06 / 2016\right)$. En su artículo 1 se dice que el objeto de la orden es: "Constituir, al amparo de lo dispuesto en el apartado segundo del artículo 7 de la Ley 4/1998, de 11 de junio, del Patrimonio Cultural Valenciano, y con funciones asesoras y consultivas, la Comisión Técnica para el Estudio e Inventario del Patrimonio Inmaterial".

35. Información facilitada por la Direcció General de Patrimoni Cultural de la Generalitat Valenciana.

36. Evidentemente, esto es simplista y reduccionista, pero con ello solo queremos presentar una radiografía que permita una lectura sencilla. Ya ha sido señalada la dificultad de otorgar a estos bienes una categoría, ver nota a pie 22 .

37. Agrupamos aquí el toque de campanas, las fallas y las tamboradas. 


\section{CONCLUSIONES}

¿Existen criterios en las declaratorias de patrimonio inmaterial en el País Valenciano? Y si los hay ¿a qué responden? En esta breve aproximación, se dibuja un mapa de claroscuros donde las razones políticas parecen tener el mayor peso, a la hora de situar y activar este patrimonio. Parece que detrás de las activaciones de los bienes no ha habido ni criterios de representatividad del territorio, ni acciones participativas ni comunitarias para su propuesta (tal y como manda la Convención de París del 2003). Además, la muestra en el País Valenciano parece indicar más bien que lo festivo, vinculado a la visión folclórica del pasado, sigue dominando en la visión del patrimonio inmaterial. Junto con ella, lo estético, por contaminación del patrimonio histórico-artístico, parece jugar un papel destacable a la hora de ser declarado BICI. Así, se han galardonado aquellos rituales que destacan por su bella factura, su espectacularidad y su escenografía. No es extraño que las grandes fiestas estén representadas por encima de otras manifestaciones culturales.

Ahora bien, en el caso valenciano, parece que la voluntad política apunta hacia altos vuelos. El interés por proponer bienes para la lista de PCI de la humanidad ha pesado más que una propuesta para articular las distintas identidades ${ }^{38}$ del territorio a partir de distinciones locales. Efectivamente, si como venimos apuntando, la reestructuración económica, con una terciarización de la economía y el desembarco de los intangibles, y la explosión del turismo han disparado el consumo de lo 'auténtico', el patrimonio inmaterial se sitúa como un bien goloso y estratégico ${ }^{39}$. El matrimonio de turismo y patrimonio se modula, en gran medida, en las cartografías glocalizadas de las mercancías de lo auténtico y lo verdadero, gracias a la puesta en marcha de estrategias de marketing heritage (mercadotecnia patrimonial) y la entrada de heritage brands (marcas de autenticidad). En esa búsqueda de nuevos valores inmateriales, frente a los materiales, $y$ en ese ansiado reencuentro con lo auténtico, el patrimonio inmaterial puede jugar un papel fundamental como motor económico. Y parece que esto se tiene claro en la esfera institucional. El título de este artículo hablaba de 'una explosión muy tangible', con él queríamos mostrar, precisamente, esta postura de la política valenciana y usarla como metáfora de la última declaración de las fiestas de las fallas como PCI, dado que las mismas se caracterizan por el uso de la pólvora. Durante los tres últimos años, la candidatura ha sido vivida como una auténtica carrera hacia la celebridad y el reconocimiento mundial, como una oportunidad de estar en el mapa. Dicho esto, también es cierto que, en los últimos años, se apunta hacia un tímido interés institucional por poner en valor el patrimonio inmaterial para circuitos locales -pensemos que veintiocho de los treinta

38. Entendemos aquí la identidad es un proceso abierto y siempre en negociación, un juego que no es sólido ni permanente (Hall 2003; Bauman 2004).

39. Para una aproximación sobre este tema, en el País Valenciano, se puede acudir a Carmona, Travé y Nogués (2015). 
bienes declarados se han producido a partir de 2010-, la propia creación hace unos meses de la Comisión Técnica para el Estudio e Inventario del Patrimonio Inmaterial, antes reseñada, parece ir encaminada en esa dirección.

Sin duda, este patrimonio también puede convertirse en una buena herramienta para vertebrar distintas identidades y sensibilidades, para fomentar el conocimiento y el respeto de la diversidad de nuestro país, más allá de dinamizar y revitalizar el sector turístico. Para ello se necesita trazar políticas culturales que redefinan los patrimonios y que los pongan en juego para la comunidad. Y esto debe pasar por establecer mecanismos reales de participación ciudadana para que la definición y construcción de lo nuestro sea realmente para nos-otros y no para los otros. Si no atendemos a ello, estaremos abocados a la fetichización, comercialización y mercantilización del patrimonio inmaterial, con todos los peligros que eso conlleva. 


\section{BIBLIOGRAFÍA}

Aikawa, Noriko (2004) “Visión histórica de la Preparación de la Convención Internacional de la UNESCO para la Salvaguardia del Patrimonio Cultural Inmaterial”, Museum International, 221:140-153.

Anderson, Benedict (1983) Imagined communities: reflections on the origins and spread of capitalism. London: Verso.

Ariño, Antonio (2002) "La expansión del patrimonio cultural", Revista de Occidente, 250: 129-150.

Bauman, Zygmunt (2001) Community: Seeking Safety in an Insecure World. Cambridge: Polity Press.

Bauman, Zygmunt (2004) Identity. Cambridge: Polity Press.

Bendix, Regina F. (2009) "Heritage between Economy and Politics: An Assessment from the Perspective of Cultural Anthropology". En Laurajane Smith and Natsuko Akagawa (ed) Intangible Heritage. London: Routledge, pp. 253-269.

Bendix, Regina F., Aditya Eggert y Arnika Peselmann, (eds.) (2012) Heritage Regimes and the State. Göttingen: Universitätsverlag Göttingen.

Boltanski, Luc y Ève Chiapello (2002) El nuevo espíritu del capitalismo. Akal: Madrid.

Bortolotto, Chiara (2011) “Le trouble du patrimoine culturel immatériel”. En Lepatrimoine culturel immatériel: enjeux d'une nouvelle catégorie, edited by Chiara Bortolotto, 21-43. Paris: Maison des Sciences de l'Homme.

Bourdieu, Pierre (1991) El sentido práctico. Madrid:Taurus.

Bourdieu, Pierre (1998): La distinción. Criterio y bases sociales del gusto. Madrid: Taurus.

Carmona, Daniel, Raúl Travé y Antonio Nogués (2015) "los misterios del patrimonio y del turismo en Elche. Lo global (UNESCO) en lo local (identidad" Revista Andaluza de Antropología,8:113-140.

Carrera Gema (2015) "La Ley 10/2015 para la Salvaguarda del PCI (2013-2014): ¿ patrimonio inmaterial o nacionalismo de Estado?” Revista ph, 88: 21-23.

Chaves, Margarita, Mauricio Montenegro y Marta Zambrano (eds) (2014) El valor del patrimonio: mercado, politicas culturales y agenciamientos sociales. Bogotá: Instituto Colombiano de Antropología e Historia.

Comaroff, John L. y Jean Comaroff (2011) Etnicidad S.A. Madrid: Katz.

Comaroff, John L., y Jean Comaroff (1991) Of Revelation and Revolution. Chicago: University of Chicago Press. 
Davallon, Jean (2010) “The Game of Heritagization”. En Xavier Roigé y Joan Frigolé (eds.), Constructing Cultural and Natural Heritage. Parks, Museums and Rural Heritage. Girona: ICRPC, pp. 27-38.

Del Mármol, Camila (2012) Pasados locales, políticas globales, Valencia, Germania-AVA. Del Mármol, Camila, Roigé, Xavier y Estrada, Ferran (2011) "Safeguarding Intangible Cultural Heritage? A critical perspective on the inventory of Intangible Cultural Heritage in Catalonia". En Lira, Sérgio; Amoêda, Rogério; Pinheiro, Cristina (coords.). Sharing Cultures 2011. Portugal, Green Lines Institute for Sustainable Development, pp. 481-490.

Del Mármol, Camila, Siniscalchi, Valeria y Estrada, Ferran (2016) "Reflecting on heritage and power: dynamics, strategies and appropriations in the Catalan Pyrenees and the French Alps". International Journal of Heritage Studies 22:341-354-

Estrada, Ferran y Del Mármol, Camila (2014) "La patrimonialización de la cultura inmaterial: los oficios". Arxius de sociologia, 30:45-58.

Franquesa, Jaume (2013) Urbanismo neoliberal, negocio inmobiliario y vida vecinal. El caso de Palma. Barcelona: Icaria.

Frigolé, Joan (2014) "Retóricas de la autenticidad en el capitalismo avanzado". Endoxa 33: 37-60.

Goldman, Michael (2001) "The birth of a discipline. Producing authoritative green knowledge World-Bank style”. Ethnography 2 (2): 191-217.

Hafstein, Valdimar (2011) "Célébrer les différences, renforcer la conformité". En Le patrimoine culturel immatériel: enjeux d'une nouvelle catégorie, edited by Chiara Bortolotto, 75-97. Paris, Maison des Sciences de l'Homme.

Hall, Stuart (2003) “Who Needs 'Identity'?". In Questions of Cultural Identity, edited by Stuart Hall and Paul Du Gay, 1-17. London: Sage.

Harrison, Rodney (2013) Heritage: critical approaches. London: Routledge.

Harvey, David (1998) La condición de la posmodernidad. Investigación sobre los orígenes del cambio cultural. Buenos Aires, Amorrurtu.

Harvey, David (2005) A brief history of neoliberalism. Oxford, Oxford University Press Harvey, David (2012) El enigma del capital. y las crisis del capitalismo, Madrid, Akal Harvey, David C (2001) "Heritage pasts and heritage presents: Temporality, meaning and the scope of heritage studies”. International Journal of Heritage Studies 7(4):319-338.

Heinich, Nathalie (2009) La fabrique du patrimoine. De la cathédrale à la petite cuillère. Paris: Maison des Sciences de l'Homme. 
Hernández, Gil M.; Beatriz Santamarina, Albert Moncusí, y María Albert (2005) La memoria construida. Patrimonio cultural y modernidad, Valencia, Tirant Lo Blanch.

Herzfeld, Michael (2004) The Body Impolitic: Artisans and Artifice in the Global Hierarchy of Value. Chicago: University of Chicago Press.

Herzfeld, Michael (2006) "Spatial cleansing. Monumental vacuity and the idea of the West". Journal of Material Culture 11(1-2): 127-149.

Herzfeld, Michael (2010) "Engagement, gentrification, and the neoliberal hijacking of history". Current Anthropology 51(2): 259-268.

Hesmondhalgh, David (2007) The cultural industries. London, Sage

Hobsbawm, Eric J. y Terence Ranger (1988) Linvent de la tradició, Vic, Eumo.

Iniesta, Montserrat (1994) Els gabinets del món. Antropologia, museus i museologies. Lleida : Pagès Editors.

Khaznadar, Chérif (2009) "Les dangers qui guettent la Convetion de 2003" en VVAA. Le patrimoine culturel immatériel à la lumière del'Extrême-Orient, Paris, Maison des cultures du monde Babel, pp.101-109.

Kirshenblatt-Gimblett, Barbara (1998) Destination Culture. Tourism, Museums, and Heritage. Berkeley: University of California Press.

Kirshenblatt-Gimblett, Barbara (2004) "Intangible Heritage as Metacultural Production". Museum international 56 (1-2): 52-65.

Kurin, Richard (2004): "Museums and Intangible Heritage: Culture Deal or Alive?", ICOM News, 4:7-9.

Kurin, Richard (2007) "Safeguarding of the Intangible Cultural Heritage: Key factors in Implementing the 2003 Convention", International Journal of Intangible Heritage 2: 1020

Lacarrrieu, Monica (2008) “Es necesario gestionar el patrimonio inmaterial? Notas y reflexiones para repensar las estrategias políticas y de gestión”, Boletín Gentión Cultural, $17: 2-26$.

Lowenthal, David (1998) El pasado es un país extraño, Barcelona, Akal.

MacCannell, Dean (1999) The Tourist: A New Theory of the Leisure Class, California: University of California Press.

MacDonald, Sharon (2013) Memorylands. Heritage and Identity in Europe Today. London: Routledge.

Martínez, Luis (2011) "La tutela legal del Patrimonio Cultural Inmaterial en España:: valoración y perspectivas". Revista de Sociales y Jurídicas, 7:123-150. 
Montenegro, Mauricio (2010) "La patrimonialización como protección contra la mercantilización: paradojas de las sanciones culturales de lo igual y lo diferente". Revista Colombiana de Antropología 46: 115-131.

Nash, Dennison (1992) "El turismo considerado como una forma de Imperialismo" en Smith, V (ed.) Anfitriones e Invitados, Endymión, Madrid, pp69-91.

Palumbo, Berardino. 2010. "Sistemi tassonomici dell'immaginario globale. Prime ipotesi di ricerca a partire dal caso Unesco". Meridiana 68: 37-72.

Pérez Galán, Beatriz (2011) "Los usos de la cultura en el discurso legislativo sobre patrimonio cultural en España. Una lectura antropológica sobre las figuras legales de protección". Revista de Antropología Experimental, 11: 11-30.

Prats, Llorenc (1997) Antropología y patrimonio. Barcelona, Ariel.

Quintero, Victoria (2003) “El patrimonio inmaterial: ¿intangible?” en Hernández, Elodia y Quintero, Victoria (coord.) Antropología y patrimonio: investigación, documentación e intervención, Sevilla, Junta de Andalucía, pp. 144-158.

Quintero, Victoria (2005) "El patrimonio intangible como instrumento para la diversidad cultural ¿una alternativa posible?" en Carrera, Gema y Dietz, Gunther (coord.), Patrimonio Inmaterial y gestión de la diversidad, Sevilla, IAPH, pp.68-84.

Roigé, Xavier y Joan Frigolé (eds.) (2010) Constructing cultural and natural heritage. Parks, museums and rural heritage. Girona: ICRPC.

Sánchez-Carretero, Cristina (2012) "Heritage Regimes and the Camino de Santiago: Gaps and Logics". En Heritage Regimes and the State, edited by Regina F. Bendix, Aditya Eggert, and Arnika Peselmann, 141-155. Göttingen: Universitätsverlag Göttingen.

Santamarina, Beatriz (2005) “Una aproximación al patrimonio cultural” en, G. Hernández, B. Santamarina, A. Moncusí y M. Albert: La memoria construida. Patrimonio cultural y modernidad, Valencia, Tirant Lo Blanch, pp. 21-51.

Santamarina, Beatriz (2013) "Los mapas geopolíticos de la Unesco: entre la distinción y la diferencia están las asimetrías. El éxito (exótico) del patrimonio inmaterial". Revista de Antropología Social 22: 263-286.

Santamarina, Beatriz y Albert Moncusí (2015) "El mercado de la autenticidad: las nuevas ficciones patrimoniales". Revista de Occidente 440-441: 93-112.

Santamarina, Beatriz, Hernández, Gil y Albert Moncusí (2008) "Patrimonio etnológico e identidades en España: un estudio comparativo a través de la legislación". Revista de Antropología Experimental, 8, 207-223.

Smith, Laurajane (2004) Archaeological Theory and the Politics of Cultural Heritage. London: Routledge. 
Smith, Laurajane (2006) Uses of heritage. New York: Routledge.

Smith, Laurajane y Akagawa, (eds) (2009) Intangible Heritage, London, Routledge. 